\title{
MicroRNA expression profiles in benign prostatic hyperplasia
}

\author{
NAN ZHANG $^{1 *}$, ZHONGYI LI $^{1 *}$, FUDING BAI $^{1}, \mathrm{NA} \mathrm{JI}^{2}$, \\ YICHUN ZHENG $^{1}$, YI LI $^{1}$, JIMIN CHEN $^{1}$ and XIAWA MAO ${ }^{1}$ \\ Departments of ${ }^{1}$ Urology and ${ }^{2}$ Anesthesia, The Second Affiliated Hospital, \\ Zhejiang University School of Medicine, Hangzhou, Zhejiang 310009, P.R. China
}

Received June 29, 2017; Accepted November 17, 2017

DOI: $10.3892 / \mathrm{mmr} .2017 .8318$

\begin{abstract}
Although alterations in microRNA (miRNA) expression have been previously investigated prostate cancer, the expression of miRNAs specifically in benign prostate hyperplasia $(\mathrm{BPH})$ of the prostatic stroma remains to be fully elucidated. In the present study, miRNAs and gene expression profiles were investigated using microarray analysis and reverse transcription quantitative-polymerase chain reaction ( $\mathrm{RT}-\mathrm{qPCR}$ ) in $\mathrm{BPH}$ tissue to clarify the associations between miRNA expression and target genes. Prostate tissue samples from five patients with $\mathrm{BPH}$ and five healthy men were analyzed using human Affymetrix miRNA and mRNA microarrays and differentially expressed miRNAs were validated using RT-qPCR with $30 \mathrm{BPH}$ and 5 healthy control samples. A total of 8 miRNAs, including miRNA (miR)-96-5p, miR-1271-5p, miR-21-3p, miR-96-5p, miR-181a-5p, miR-143-3p, miR-4428 and miR-106a-5p were upregulated and 8 miRNAs (miR-16-5p, miR-19b-5p, miR-940, miR-25, miR-486-3p, miR-30a-3p, let-7c and miR-191) were downregulated. Additionally, miR-96-5p was demonstrated to have an inhibitory effect on the mRNA expression levels of the following genes: Mechanistic target of rapamycin (MTOR), RPTOR independent companion of MTOR complex 2, syntaxin 10, autophagy-related protein 9A, zinc finger E-box binding homeobox 1, caspase 2 and protein kinase $\mathrm{c} \varepsilon$. Additionally, 16 differentially expressed miRNAs were identified using RT-qPCR analysis. This preliminary study provides a solid basis for a further functional study to investigate the underlying regulatory mechanisms of $\mathrm{BPH}$.
\end{abstract}

Correspondence to: Dr Xiawa Mao or Dr Nan Zhang, Department of Urology, The Second Affiliated Hospital, Zhejiang University School of Medicine, 88 Jiefang Road, Hangzhou, Zhejiang 310009, P.R. China

E-mail:mao_xiawa@zju.edu.cn

E-mail: nanzhang@zju.edu.cn

*Contributed equally

Key words: microRNA, benign prostatic hyperplasia, microRNA-96-5p

\section{Introduction}

Benign prostate hyperplasia (BPH) is one of the most common diseases in the aging male population that seriously affects patients' quality of life (1). It is estimated that BPH occurs in $30-60 \%$ of men $\geq 50$ years in mainland China, with the disease incidence increasing with age (2). In an aging population, this disease is emerging as an important clinical topic. Although a wide variety of drugs may be used to treat $\mathrm{BPH}$, there are limitations in the application of these drugs due to drug dependence and severe side effects $(3,4)$. A previous study demonstrated that microRNA (miRNA) dysregulation is involved in the development and progression of $\mathrm{BPH}$ (5). However, the exact involvement of miRNAs in BPH is not fully understood, due to the complex interaction between miRNAs and gene expression levels.

miRNAs are single-stranded non-coding RNAs of $\sim 22$ nucleotides (6) that control gene expression at the post-transcriptional level by mRNA cleavage or translational suppression (6). Currently, there are 2,603 human mature miRNAs registered at miRNA database miRBase release 21.0, and the number of newly discovered miRNAs is gradually increasing. Previous studies demonstrated that miRNAs have key regulatory roles in numerous biological processes, including development, differentiation, signal transduction and cell maintenance $(7,8)$. miRNA dysregulation is also a common feature of various diseases, including cancer, inflammation, autoimmune disease and prostate hyperplasia $(9,10)$.

To date, despite several studies reporting that miRNA mediates prostate cancer, few articles have investigated miRNA regulation in BPH (11-13). To the best of our knowledge, only two studies have examined miRNA expression in BPH and healthy controls, which provided inconsistent findings $(14,15)$. Furthermore, limited microarray data has been confirmed by reverse transcription-quantitative polymerase chain reaction (RT-qPCR), and only one previous study has reported an association between Homo sapiens (hsa)-miR-143 or hsa-miR-145 and clinicopathological data in BPH (14). In the present study, miRNA and gene expression profiles in prostate tissue of patients with BPH and healthy men were investigated using microarray analysis and RT-qPCR.

\section{Materials and methods}

Patients and tissue samples. Prostate tissue samples were collected from 5 patients with BPH and 5 healthy volunteers 
(aged $42 \pm 10$ years) at The Second Affiliated Hospital,Zhejiang University School of Medicine (Hangzhou, China) between December 2016 and January 2017. The participants included in the current study had not received any medical treatment prior to tissue collection. A total of 10 prostate tissue samples were immediately snap-frozen in liquid nitrogen following radical prostatectomy. Extracted RNA was used for microarray analyses and RT-qPCR. An additional $25 \mathrm{BPH}$ samples (aged $43 \pm 8$ years) were included in the RT-qPCR assay between January 2017 and April 2017 at The Second Affiliated Hospital, Zhejiang University School of Medicine. Clinicopathological information for each patient was obtained, including age and levels of preoperative prostate-specific antigen. The present study was conducted with the approval of The Ethics Review Board at Zhejiang University School of Medicine (Hangzhou, China) and all participants provided written informed consent.

Cell culture. WPMY-1 cells obtained from the cell bank at the Chinese Academy of Sciences (Shanghai, China) were cultured in DMEM medium (11965-092; Gibco; Thermo Fisher Scientific, Inc., Waltham, MA, USA) containing $100 \mathrm{U} / \mathrm{ml}$ penicillin/streptomycin and $10 \%$ fetal bovine serum (15140-122 and 10099-141 both from Gibco; Thermo Fisher Scientific, Inc.) at $37^{\circ} \mathrm{C}$ in $5 \% \mathrm{CO}_{2}$, as previously described (16).

RNA extraction. RNA was extracted with the miRNeasy Mini kit (Qiagen GmbH, Hilden, Germany) according to the manufacturer's protocol. A NanoDrop ND-1000 spectrometer (Thermo Fisher Scientific, Inc., Pittsburgh, PA, USA) was used to detect the RNA yield and 260/280 nm ratio. A 2100 Bioanalyzer (Agilent Technologies, Inc., Santa Clara, CA, USA) was used to quantify the RNA integrity number.

miRNA and mRNA microarray experiments. Differential expression analysis of miRNAs and mRNA between the pooled samples was performed using Affymetrix MiRNA Microarray technology version 4.0 (Affymetrix; Thermo Fisher Scientific, Inc.) (17) and GeneChip Human Transcriptome Array 2.0 (Thermo Fisher Scientific, Inc.) $(18,19)$.

miRNA RT-qPCR. A TaqMan miRNA assay (Thermo Fisher Scientific, Inc.) was used to detect mature miRNAs using a Bio-Rad IQ5 (Bio-Rad Laboratories, Inc., Hercules, CA, USA) according to the manufacturer's protocol, as previously described (6). The reactions were performed using the following parameters: $95^{\circ} \mathrm{C}$ for 2 min followed by 40 cycles of $95^{\circ} \mathrm{C}$ for $15 \mathrm{sec}$ and $60^{\circ} \mathrm{C}$ for $30 \mathrm{sec}$. RNU6-1 small nuclear RNA was used as an endogenous control for data normalization. Relative expression was calculated using the comparative threshold cycle method (20). Experiments were performed in triplicate. RT-qPCR analyses for the mRNA expression of target genes were performed using PrimeScript RT-PCR kits (Takara Biotechnology Co., Ltd., Dalian, China). The reactions were performed using the following parameters: $95^{\circ} \mathrm{C}$ for $2 \mathrm{~min}$ followed by 40 cycles of $95^{\circ} \mathrm{C}$ for $30 \mathrm{sec}, 60^{\circ} \mathrm{C}$ for $30 \mathrm{sec}, 72^{\circ} \mathrm{C}$ for $20 \mathrm{sec}$, and then $72^{\circ} \mathrm{C}$ for $10 \mathrm{~min}$. $\beta$-actin was used as an internal control (20). The primer sequences used are presented in Table I.
Table I. Primer sequences used in reverse transcription quantitative-polymerase chain reaction.

\begin{tabular}{lcl}
\hline Gene & Direction & \multicolumn{1}{c}{ Primer sequence (5'- 3') } \\
\hline MTOR & F & ACAGCCCAGGCCGCATTGTC \\
& R & TCCAGGGACTCCGTCAGGCG \\
RICTOR & F & TCCGAAGAAATGTCAGTCAG \\
& R & GGCAGTGGCACAAGTGTT \\
STX10 & F & ATCCCACATGACGAGTGGTG \\
& R & GGTCTGGGAACGAGAAGGTC \\
GRB2 & F & CCAGAGCCAAGGCAGAAGAA \\
& R & AGTACTTCCCGGCTCCATCT \\
ATG9A & F & CGTGTGGGAAGGACAG \\
& R & GGCGCTTTCTCCACTC \\
ZEB1 & F & TTAGTTGCTCCCTGTGCAGTT \\
& R & TAGGAGCCAGAATGGGAAAAG \\
CASP2 & F & TCCAGCACAAGGAGCTGATG \\
PRKCE & R & GCTAGCACCACTCGGTTCTT \\
& F & CACTGCAGTTGGACTTTGCC \\
ACTB & R & GACAGTGGACATGGGACTGG \\
& F & TTCCTTCCTGGGCATGGAGTCC \\
& R & TGGCGTACAGGTCTTTGCGG \\
\hline
\end{tabular}

F, forward; R, reverse; MTOR, mechanistic target of rapamycin; RICTOR, RPTOR independent companion of MTOR complex 2; STX10, syntaxin 10; GRB2, growth factor receptor bound protein 2; ATG9A, autophagy-related protein 9A; ZEB1, zinc finger E-box binding homeobox 1; CASP2, caspase-2; PRKCE, protein kinase $\mathrm{c} \varepsilon$; ACTB, $\beta$ actin.

Gene ontology (GO) enrichment and Kyoto Encyclopedia of GenesandGenomes (KEGG) pathwayanalysis.Inordertofurther understand the functions of miRNA and their targets, GO enrichment and KEGG pathway analysis for all annotated miRNAs and their targets were performed. Blast2GO was employed to store information from the GO (http://www.geneontology.org/) and KEGG (http://www.kegg.jp) pathway databases. All the sequences were identified by BLASTx searches against the GO protein database. A combined query was used in order to complete the GO annotation and pathway analysis against the GO and KEGG databases (21).

Transfection experiment. Mimic and control miR-96-5p were purchased from Shanghai GenePharma Co., Ltd. (Shanghai, China) and transfections were performed with DharmaFECT 1 transfection reagent (Thermo Fisher Scientific, Inc.). WPMY-1 cells were transfected with $100 \mathrm{nM}$ mimic or miRNA control for $48 \mathrm{~h}$, and then mRNA expression was subsequently assessed by RT-qPCR.

Statistical analysis. Data are expressed as the mean \pm standard error of the mean. Statistical analysis was performed using the Student's t-test for comparison of two groups in microarray analysis, and analysis of variance for comparisons of expression of selected aberrant miRNAs in RT-qPCR assay, using SPSS version 17.0 (SPSS, Inc., Chicago, IL, USA). P<0.05 was considered to indicate a statistically significant difference. 


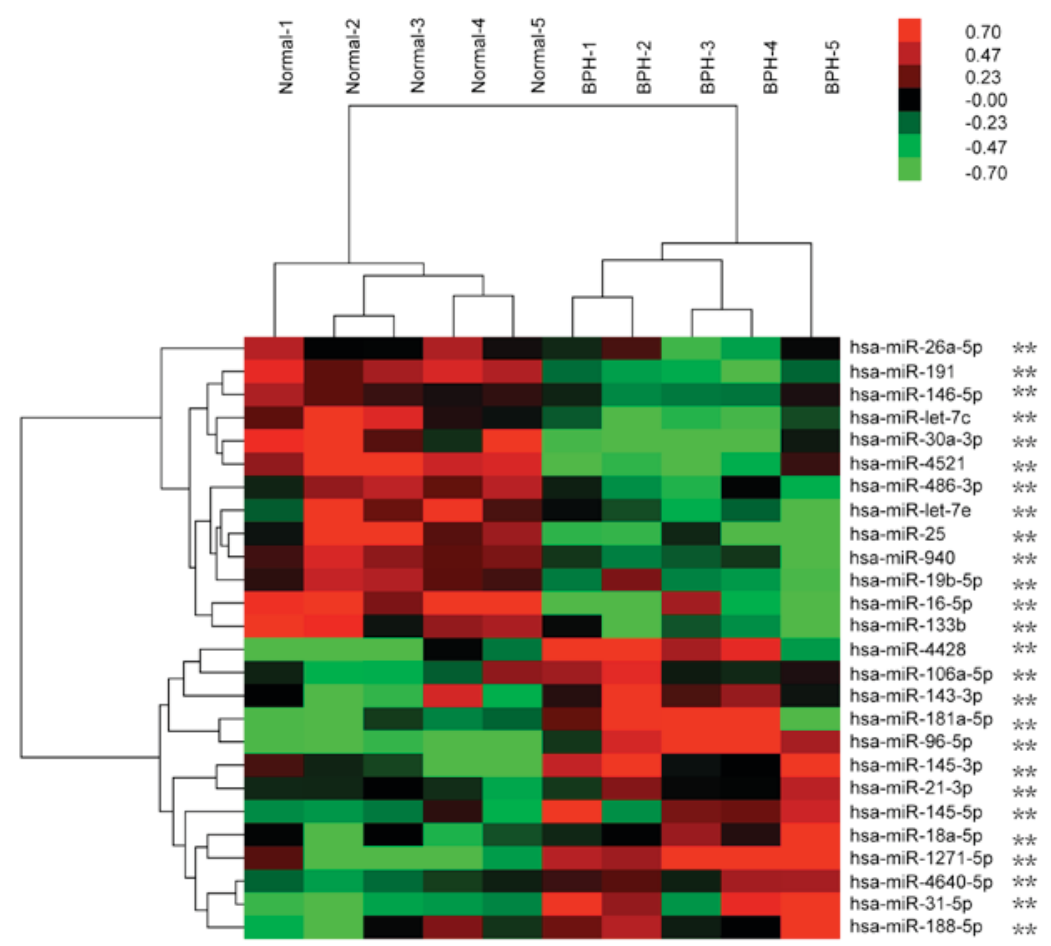

Figure 1. Differentially expressed miRNAs in the BPH and control groups. miRNA profiles of prostates differentiate between 5 patients with BPH and 5 healthy men. Both downregulated (red) and upregulated (green) miRNAs were identified ( $\left.{ }^{* *} \mathrm{P}<0.001\right)$. miRNA, microRNA; BPH, benign prostatic hyperplasia; hsa-miR, Homo sapiens microRNA.

\section{Results}

miRNA expression profiles in the prostate of patients with $B P H$ and healthy controls. To investigate the miRNA expression profiles in the BPH and control group, array-based miRNA profiling of the prostate tissues from patients with BPH was performed. Of the 1,872 human miRNAs assayed, 23 miRNAs including miR-4428, miR-106a-5p, miR-96-5p and miR-1271-5p had higher expression levels in the prostate of patients with BPH when compared with healthy controls. The expression levels of 15 miRNAs including miR-16-5p, miR-19b-5p, miR-940, and miR-25 were reduced in the prostate of patients with BPH compared with healthy controls (Fig. 1). Of these 26 miRNAs, miR-96-5p and miR-1271-5p were significantly overexpressed, whereas miR-30a was significantly downregulated in the prostates of men with BPH.

Validation of miRNA dysregulation by miRNA RT-qPCR analysis. To confirm the microarray findings, the relative expression of each dysregulated miRNA was examined with miRNA RT-qPCR in all $30 \mathrm{BPH}$ samples and the 5 healthy controls. The expression levels of hsa-miR-96-5p, hsa-miR-1271-5p, hsa-miR-21-3p, hsa-miR-96-5p, hsa-miR-181a-5p, hsa-miR-143-3p, hsa-miR-4428 and hsa-miR-106a-5p were significantly upregulated in the BPH group compared with healthy controls. The expression levels of hsa-miR-16-5p, hsa-miR-19b-5p, hsa-miR-940, hsa-miR-25, hsa-miR-486-3p, hsa-miR-30a-3p, hsa-miR-let-7c and hsa-miR-191 were significantly downregulated compared with the control (Fig. 2). These findings were in concordance with the microarray assay results. Notably, hsa-miR-96-5p
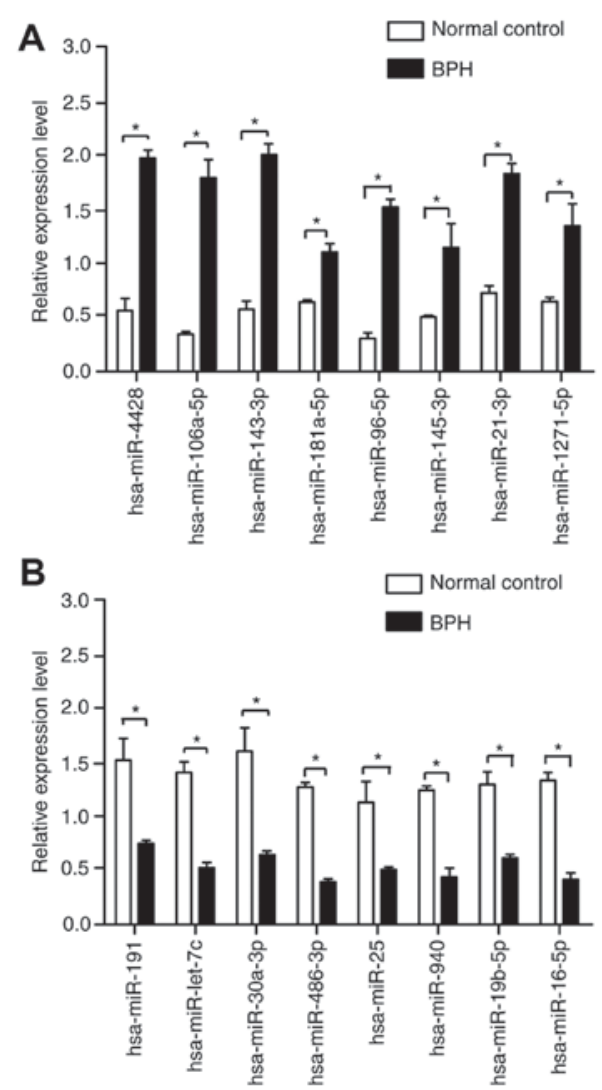

Figure 2. Validation of microarray data with miRNA reverse transcription quantitative-polymerase chain reaction. Expression levels of miRNAs were detected in $30 \mathrm{BPH}$ cases and 5 healthy cases. (A) miRNAs with upregulated or (B) downregulated expression compared with the control group. ${ }^{*} \mathrm{P}<0.05$; miRNA, microRNA; BPH, benign prostatic hyperplasia; hsa-miR, Homo sapiens microRNA. 


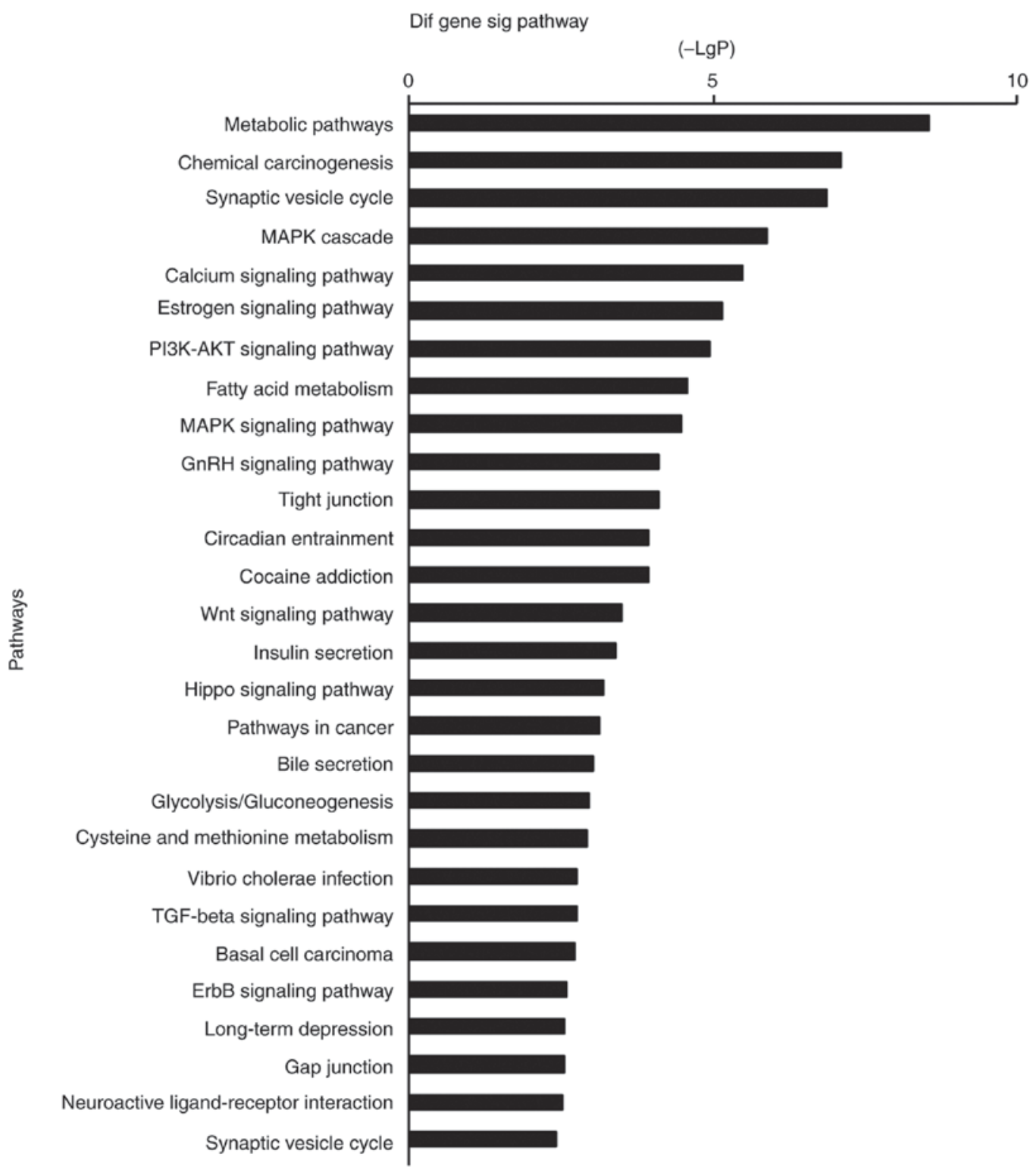

Figure 3. Pathway analysis based on miRNA target genes. The significant pathways downregulated by miRNAs are presented. -LgP is the negative logarithm of the P-value, with a larger - $\mathrm{LgP}$ indicating a smaller P-value. The pathways were likely upregulated due to the dysregulation of miRNAs in BPH. miRNA, microRNA; BPH, benign prostatic hyperplasia; MAPK, mitogen-activated protein kinase; PI3K, phosphoinositide 3-kinase; AKT, protein kinase B; GnRH, gonadotropin-releasing hormone; TGF- $\beta$, transforming growth factor $\beta$; ErbB, epidermal growth factor receptor.

and hsa-miR-1271-5p have the same seed sequence and were classified in the same cluster.

Bioinformatics analysis. Gene ontology and KEGG pathway analysis of the signaling pathways regulated by miRNAs (as identified by RT-qPCR) revealed 47 upregulated signaling pathways and 28 downregulated signaling pathways in the BPH group compared to healthy controls. These regulated signaling pathways were involved in numerous cellular processes, including metabolic (pyrimidine, glutathione, phenylalanine, gluconeogenesis, and pyruvate), oncogenic (prostate cancer, colorectal cancer, and bladder cancer) and adhesive and signal transduction [estrogen, phosphoinositide 3-kinase (PI3K-AKT), Wnt, transforming growth factor $\beta$ (TGF- $\beta$ ) and mitogen-activated protein kinase (MAPK)] signaling pathways. In addition, according to the enrichments of the significantly downregulated signal genes, there were 28 downregulated signaling pathways (Fig. 3).

The miRNA-gene network of overlapping target genes identified the involvement of hsa-miR-96-5p (Fig. 4A). The major target genes of hsa-miR-96-5p were identified by TargetScan version 7.1 (www.targetscan.org), include mechanistic target of rapamycin (MTOR), RPTOR independent companion of MTOR complex 2 (RICTOR), syntaxin 10 (STX10), growth receptor bound protein 2 (GRB2), autophagy related 9A, zinc finger E-box binding homeobox 1 (ZEB1), caspase 2 (CASP2) and protein kinase $\mathrm{c} \varepsilon$ (PRKCE). In order to determine whether these genes were miR-96 target genes, WPMY-1 cells were transfected with miR-96-5p mimics or miR-96-5p control. As presented in Fig. 4B, miR-96 had an inhibitory effect on the mRNA expression level of these target genes, excluding GRB2. This verified that miR-96 may downregulate these target genes. 
A

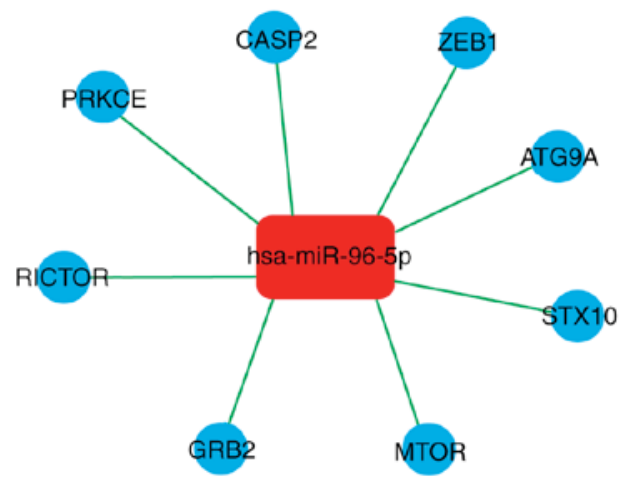

B

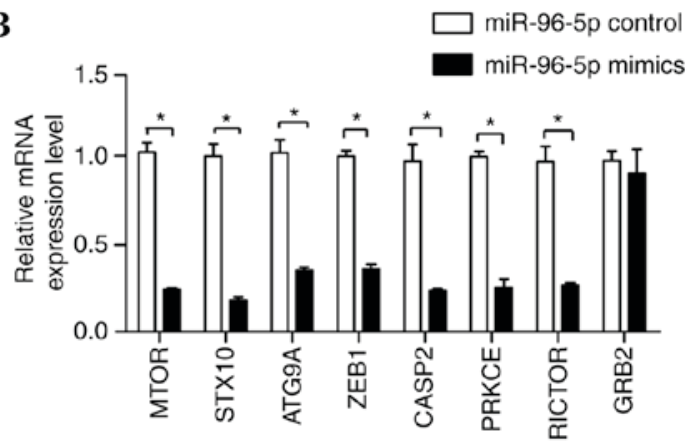

Figure 4. Major target genes of hsa-miR-96-5p. (A) miRNA-gene network. Red and blue box nodes represent upregulated miR-96-5p and downregulated genes, respectively. Edges represent the inhibitory effect of the miRNA on gene expression. (B) WPMY-1 cells were transfected with $100 \mathrm{nM}$ miR-96-5p mimic or control at $48 \mathrm{~h}$. The mRNA levels of these target genes were determined by reverse transcription quantitative-polymerase chain reaction. ${ }^{*} \mathrm{P}<0.05$. miRNA, microRNA; hsa-miR, Homo sapiens microRNA; MTOR, mechanistic target of rapamycin; STX10, syntaxin 10; ATG9A, autophagy-related protein 9A; ZEB1, zinc finger E-box binding homeobox 1; CASP2, caspase-2; PRKCE, protein kinase c $\varepsilon$; RICTOR, RPTOR independent companion of MTOR complex 2; GRB2, growth factor receptor-bound protein 2 .

\section{Discussion}

miRNAs may regulate gene expression in various biological processes, including cancer, development, differentiation, signal transduction and cell maintenance $(22,23)$. However, there are limited studies regarding differential miRNA expression in BPH. In the present study, miRNA expression profiles in the BPH and control group were evaluated to identify advanced BPH signatures. RT-qPCR was also used to identify some differentially expressed miRNAs. These findings are of note for future studies on BPH as miRNAs are a major determinant of cell proliferation.

The results of microarray analysis in the prostate specimens of $5 \mathrm{BPH}$ and 5 healthy men revealed that 26 miRNAs were dysregulated. Among these differentially expressed miRNAs, miR-106a, miR-143, miR-96 and miR-1271 were overexpressed in the BPH group compared with controls, whereas the remaining 13 miRNAs were downregulated. A total of 8 upregulated miRNAs and 8 downregulated miRNAs were identified by RT-qPCR, suggesting that aberrantly expressed miRNAs may be involved in BPH development.

Altered miR-143, miR-106, miR-181, miR-21, miR-30a and miR-16 expression has been previously demonstrated to be involved in numerous types of cancer, including pancreatic,

colorectal, prostate and breast cancer (24-29). The microarray profile data of the current study revealed that these 6 miRNAs were also dysregulated in the BPH group, which may indicate their contribution to the pathogenesis of BPH and prostate cancer. Additionally, Viana et al (14) reported that miR-143 and miR-145 may be involved in the pathogenesis of BPH by inhibiting target genes, that include MAP kinase kinase kinase kinase 4, KRAS proto-oncogene, GTPase and MAP kinase kinase kinase 3. Consistent with these findings, miR-143 was overexpressed in the BPH group compared with controls in the present study. Therefore, overexpression of miR-143 may contribute to $\mathrm{BPH}$ and further study of miR-143 dysregulation may provide an insight into the disease pathogenesis.

The miR-96-1271 cluster has been previously demonstrated to have different roles in various disease processes and may act as an oncogene or a tumor suppressor $(30,31)$. Fendler et al (32) identified that dysregulation of the miR-96 cluster in prostate cancer resulted in forkhead box $\mathrm{O} 1$ inhibition, demonstrating its function as an oncogene. In the present study, several target genes of the miR-96-1271 cluster were predicted in silico, including MTOR, STX10, RICTOR, ZEB1, CASP2 and PRKCE. To date, many genes have been experimentally validated as targets of the miR-96-1271 cluster, including receptors, transcriptional regulatory proteins, kinases and oncogenes (33-36). In the present study, WPMY-1 cells were transfected with miR-96-5p mimic or control and the mRNA expression of these genes was detected with RT-qPCR. MiR-96 was demonstrated to have an inhibitory effect on the mRNA expression level of these target genes, excluding GRB2.

Gene ontology enrichment analysis identified target genes that were differentially regulated by miRNAs and also had different functions in biological processes between the BPH and control group, including cell proliferation, differentiation, adhesion, cycle, apoptosis and metabolism. In the current study, 47 upregulated and 28 downregulated pathways were identified to have potential involvement in BPH development. These signal pathways were abundant among the significantly enriched pathways, including metabolic (pyruvate, phenylalanine, and gluconeogenesis), oncogenic (prostate, colorectal and bladder cancer), and adhesive and signaling (estrogen, PI3K-AKT, Wnt, TGF- $\beta$ and MAPK) pathways. These results demonstrate that miRNAs may influence several signaling pathways that affect BPH progression.

In summary, dysregulated miRNA expression may be involved in the progression of BPH through miRNA target gene inhibition. The specific mechanism of miRNA involvement in BPH remains to be determined. However, the present study provides a foundation for further study of the role of miRNAs in BPH.

\section{Acknowledgements}

The current study was supported by a grant from The National Natural Science Foundation of China (grant nos. 81400756 and 81402099), the projects of the Medical and Health Technology Development Program in Zhejiang Province (grant no. 2016147031) and The Public Welfare Technology Project of Science Technology Department of Zhejiang Province (grant no. 2017C33063). 


\section{References}

1. Cindolo L, Alvarez-Maestro M, Castellucci R and Schips L: Efficacy and safety of dutasteride for the treatment of symptomatic benign prostatic hyperplasia (BPH): A systematic review and meta-analysis. World J Urol 33: 441-442, 2015.

2. Kaplan SA: Re: Influence of immune inflammation on androgen receptor expression in benign prostatic hyperplasia tissue. J Urol 189: 1002, 2013.

3. Gacci M, Salvi M, Sebastianelli A, Vignozzi L, Corona G, McVary KT, Kaplan SA, Maggi M, Carini M and Oelke M: The use of a single daily dose of tadalafil to treat signs and symptoms of benign prostatic hyperplasia and erectile dysfunction. Res Rep Urol 5: 99-111,2013.

4. Gao YA, Huang Y, Zhang R, Yang YD, Zhang Q, Hou M and Wang Y: Benign prostatic hyperplasia: Prostatic arterial embolization versus transurethral resection of the prostate-a prospective, randomized and controlled clinical trial. Radiology 270:920-928, 2014.

5. Cochetti G, Poli G, Guelfi G, Boni A, Egidi MG and Mearini E: Different levels of serum microRNAs in prostate cancer and benign prostatic hyperplasia: Evaluation of potential diagnostic and prognostic role. Onco Targets Ther 9: 7545-7553, 2016.

6. Tang B, Li N, Gu J, Zhuang Y, Li Q, Wang HG, Fang Y, Yu B, Zhang JY, Xie QH, et al: Compromised autophagy by MIR30B benefits the intracellular survival of Helicobacter pylori. Autophagy 8: 1045-1057, 2012.

7. Andersen GB, Knudsen A, Hager H, Hansen LL and Tost J: miRNA profiling identifies deregulated miRNAs associated with osteosarcoma development and time to metastasis in two large cohorts. Mol Oncol: Nov 9, 2017 (Epub ahead of print).

8. Elliott KJ, Bourne AM, Takayanagi T, Takaguri A, Kobayashi T, Eguchi K and Eguchi S: ADAM17 silencing by adenovirus encoding miRNA-embedded siRNA revealed essential signal transduction by angiotensin II in vascular smooth muscle cells. J Mol Cell Cardiol 62: 1-7, 2013.

9. Bordinhao AL, Evangelista AF, Oliveira RJ, Macedo T, Silveira HC, Reis RM and Marques MM: MicroRNA profiling in human breast cancer cell lines exposed to the anti-neoplastic drug cediranib. Oncol Rep 36: 3197-3206, 2016.

10. Chen P, Zhao H, Huang J, Yan X, Zhang Y and Gao Y: MicroRNA-17-5p promotes gastric cancer proliferation, migration and invasion by directly targeting early growth response 2 . Am J Cancer Res 6: 2010-2020, 2016.

11. Fu Y and Cao F: MicroRNA-125a-5p regulates cancer cell proliferation and migration through NAIF1 in prostate carcinoma. Onco Targets Ther 8: 3827-3835, 2015.

12. Chen WY, Liu SY, Chang YS, Yin JJ, Yeh HL, Mouhieddine TH, Hadadeh O, Abou-Kheir W and Liu YN: MicroRNA-34a regulates WNT/TCF7 signaling and inhibits bone metastasis in Ras-activated prostate cancer. Oncotarget 6: 441-457, 2015.

13. Katz B, Reis ST, Viana NI, Morais DR, Moura CM, Dip N, Silva IA, Iscaife A, Srougi M and Leite KR: Comprehensive study of gene and microRNA expression related to epithelial-mesenchymal transition in prostate cancer. PLoS One 9: e113700, 2014.

14. Viana NI, Reis ST, Dip NG, Morais DR, Moura CM, Silva IA Katz B, Srougi M, Leite KR and Antunes AA: MicroRNAs 143 and 145 may be involved in benign prostatic hyperplasia pathogenesis through regulation of target genes and proteins. Int J Biol Markers 29: e246-252, 2014.

15. Haj-Ahmad TA, Abdalla MA and Haj-Ahmad Y: Potential urinary miRNA biomarker candidates for the accurate detection of prostate cancer among benign prostatic hyperplasia patients. J Cancer 5: 182-191, 2014.

16. Zhang N, Ji N, Jiang WM, Li ZY, Wang M, Wen JM, Li Y, Chen $X$ and Chen JM: Hypoxia-induced autophagy promotes human prostate stromal cells survival and ER-stress. Biochem Biophys Res Commun 464: 1107-1112, 2015.

17. Wu D and Gantier MP: Normalization of Affymetrix miRNA microarrays for the analysis of cancer samples. Methods Mol Biol 1375: 1-10, 2016.

18. Feber A, Xi L, Pennathur A, Gooding WE, Bandla S, Wu M, Luketich JD, Godfrey TE and Litle VR: MicroRNA prognostic signature for nodal metastases and survival in esophageal adenocarcinoma. Ann Thorac Surg 91: 1523-1530, 2011.
19. Smeets A, Daemen A, Vanden Bempt I, Gevaert O, Claes B, Wildiers H, Drijkoningen R, Van Hummelen P, Lambrechts D, De Moor B, et al: Prediction of lymph node involvement in breast cancer from primary tumor tissue using gene expression profiling and miRNAs. Breast Cancer Res Treat 129: 767-776, 2011.

20. Livak KJ and Schmittgen TD: Analysis of relative gene expression data using real-time quantitative PCR and the 2(-Delta Delta C(T)) method. Methods 25: 402-408, 2001.

21. Gao F, Nan F, Feng J, Lv J, Liu Q and Xie S: Identification and characterization of microRNAs in Eucheuma denticulatum by high-throughput sequencing and bioinformatics analysis. RNA Biol 13: 343-352, 2016.

22. Hayashi K, Chuva de Sousa Lopes SM, Kaneda M, Tang F, Hajkova P, Lao K, O'Carroll D, Das PP, Tarakhovsky A, Miska EA and Surani MA: MicroRNA biogenesis is required for mouse primordial germ cell development and spermatogenesis. PLoS One 3: e1738, 2008.

23. Zhang C: MicroRNomics: A newly emerging approach for disease biology. Physiol Genomics 33: 139-147, 2008.

24. Su J, Liang H, Yao W, Wang N, Zhang S, Yan X, Feng H, Pang W, Wang Y, Wang X, et al: MiR-143 and MiR-145 regulate IGF1R to suppress cell proliferation in colorectal cancer. PLoS One 9: e114420, 2014

25. Xu YF, Li YQ, Guo R, He QM, Ren XY, Tang XR, Jia WH, Kang TB, Zeng MS, Sun Y, et al: Identification of miR-143 as a tumour suppressor in nasopharyngeal carcinoma based on microRNA expression profiling. Int $\mathrm{J}$ Biochem Cell Biol 61: 120-128, 2015.

26. Zhang GJ, Li JS, Zhou H, Xiao HX, Li Y and Zhou T: MicroRNA-106b promotes colorectal cancer cell migration and invasion by directly targeting DLC1. J Exp Clin Cancer Res 34: 73, 2015.

27. Huang P, Ye B, Yang Y, Shi J and Zhao H: MicroRNA-181 functions as a tumor suppressor in non-small cell lung cancer (NSCLC) by targeting Bcl-2. Tumour Biol 36: 3381-3387, 2015.

28. Khan K, Cunningham D, Peckitt C, Barton S, Tait D, Hawkins M, Watkins D, Starling N, Rao S, Begum R, et al: miR-21 expression and clinical outcome in locally advanced pancreatic cancer: Exploratory analysis of the pancreatic cancer Erbitux, radiotherapy and UFT (PERU) trial. Oncotarget 7: 12672-12681, 2016.

29. Cheng CW, Wang HW, Chang CW, Chu HW, Chen CY, Yu JC, Chao JI, Liu HF, Ding SL and Shen CY: MicroRNA-30a inhibits cell migration and invasion by downregulating vimentin expression and is a potential prognostic marker in breast cancer. Breast Cancer Res Treat 134: 1081-1093, 2012.

30. Haflidadottir BS, Larne O, Martin M, Persson M, Edsjö A, Bjartell A and Ceder Y: Upregulation of miR-96 enhances cellular proliferation of prostate cancer cells through FOXO1. PLoS One 8: e72400, 2013

31. Ress AL, Stiegelbauer V, Winter E, Schwarzenbacher D, Kiesslich T, Lax S, Jahn S, Deutsch A, Bauernhofer T, Ling H, et al: MiR-96-5p influences cellular growth and is associated with poor survival in colorectal cancer patients. Mol Carcinog 54: 1442-1450, 2015.

32. Fendler A, Jung M, Stephan C, Erbersdobler A, Jung K and Yousef GM: The antiapoptotic function of miR-96 in prostate cancer by inhibition of FOXO1. PLoS One 8: e80807, 2013.

33. Assal RA, El Tayebi HM, Hosny KA, Esmat G and Abdelaziz AI: A pleiotropic effect of the single clustered hepatic metastamiRs miR-96-5p and miR-182-5p on insulin-like growth factor II, insulin-like growth factor-1 receptor and insulin-like growth factor-binding protein-3 in hepatocellular carcinoma. Mol Med Rep 12: 645-650, 2015.

34. Vishwamitra D, Li Y, Wilson D, Manshouri R, Curry CV, Shi B, Tang XM, Sheehan AM, Wistuba II, Shi P and Amin HM: MicroRNA 96 is a post-transcriptional suppressor of anaplastic lymphoma kinase expression. Am J Pathol 180: 1772-1780, 2012.

35. Wu Z, Liu K, Wang Y, Xu Z, Meng J and Gu S: Upregulation of microRNA-96 and its oncogenic functions by targeting CDKN1A in bladder cancer. Cancer Cell Int 15: 107, 2015.

36. Yu S, Lu Z, Liu C, Meng Y, Ma Y, Zhao W, Liu J, Yu J and Chen J: miRNA-96 suppresses KRAS and functions as a tumor suppressor gene in pancreatic cancer. Cancer Res 70: 6015-6025, 2010 . 